\title{
Chebyshev Type Integral Inequalities Involving the Fractional Hypergeometric Operators
}

\author{
D. Baleanu ${ }^{1,2,3}$ and S. D. Purohit ${ }^{4}$ \\ ${ }^{1}$ Department of Chemical and Materials Engineering, Faculty of Engineering, King Abdulaziz University, P.O. Box 80204, \\ Jeddah 21589, Saudi Arabia \\ ${ }^{2}$ Department of Mathematics and Computer Sciences, Faculty of Arts and Sciences, Cankaya University, 06530 Ankara, Turkey \\ ${ }^{3}$ Institute of Space Sciences, Magurele, Bucharest, Romania \\ ${ }^{4}$ Department of Basic Sciences (Mathematics), College of Technology and Engineering, \\ M.P. University of Agriculture and Technology, Udaipur 313001, India
}

Correspondence should be addressed to S. D. Purohit; sunil_a_purohit@yahoo.com

Received 31 January 2014; Accepted 8 March 2014; Published 22 April 2014

Academic Editor: Juan J. Nieto

Copyright (C) 2014 D. Baleanu and S. D. Purohit. This is an open access article distributed under the Creative Commons Attribution License, which permits unrestricted use, distribution, and reproduction in any medium, provided the original work is properly cited.

By making use of the fractional hypergeometric operators, we establish certain new fractional integral inequalities for synchronous functions which are related to the weighted version of the Chebyshev functional. Some consequent results and special cases of the main results are also pointed out.

\section{Introduction}

Fractional integral inequalities have proved to be one of the most important and powerful tools for the development of many branches of pure and applied mathematics. These inequalities have many applications in numerical quadrature, transform theory, probability, and statistical problems, but the most useful ones are in establishing uniqueness of solutions in fractional boundary value problems. Moreover, they also provide upper and lower bounds to the solutions of the above equations. Therefore, a significant development in the classical and fractional integral inequalities, particularly in analysis, has been witnessed; see, for instance, the papers [1-7] and the references cited therein.

Let the functional

$$
\begin{aligned}
T(f, g)= & \frac{1}{b-a} \int_{a}^{b} f(x) g(x) d x \\
& -\left(\frac{1}{b-a} \int_{a}^{b} f(x) d x\right)\left(\frac{1}{b-a} \int_{a}^{b} g(x) d x\right),
\end{aligned}
$$

where $f$ and $g$ are two integrable functions and synchronous on $[a, b]$; that is,

$$
(f(x)-f(y))(g(x)-g(y)) \geq 0,
$$

for any $x, y \in[a, b]$; then the Chebyshev integral inequality [8] is given by $T(f, g) \geq 0$. The sign of this inequality is reversed if $f$ and $g$ are asynchronous on $[a, b]$ (i.e., $(f(x)-$ $f(y))(g(x)-g(y)) \leq 0$, for any $x, y \in[a, b])$. Under various assumptions (Chebyshev inequalities, Grüss inequality, etc.) [9-11], Chebyshev functionals are useful to give a lower bound or an upper bound for $T(f, g)$, in the theory of approximations. Therefore, in the literature, we found several papers that analyze extensions and generalizations of these integral inequalities by involving fractional calculus and $q$ calculus operators. One may refer to such type of works in the book [12] and the recent papers [13-24]. A similar type of important role is also played by the Chebyshev polynomials of first and second kind in the theory of approximation. For a detailed account about these polynomials and their generating functions, one can see a recent paper [25] and research monograph by Srivastava and Manocha [26]. 
For the present paper, let us consider the weighted version of the Chebyshev functional (see [8])

$$
\begin{aligned}
T(f, g, p)= & \int_{a}^{b} p(x) d x \int_{a}^{b} f(x) g(x) p(x) d x \\
& -\int_{a}^{b} f(x) p(x) d x \int_{a}^{b} g(x) p(x) d x
\end{aligned}
$$

provided that $f$ and $g$ are two integrable functions on $[a, b]$ and $p(x)$ is a positive and integrable function on $[a, b]$. In 2000, Dragomir [27] derived the following inequality:

$$
2|T(f, g, p)| \leq\left\|f^{\prime}\right\|_{r}\left\|g^{\prime}\right\|_{s}\left[\iint_{a}^{b}|x-y| p(x) p(y) d x d y\right]
$$

where $f, g$ are two differentiable functions and $f^{\prime} \in L_{r}(a, b)$, $g^{\prime} \in L_{s}(a, b), r>1, r^{-1}+s^{-1}=1$. Recently, Dahmani et al. [28] established some integral results related to Chebyshev's functional (3) in the case of differentiable functions whose derivatives belong to the space $L_{p}([0, \infty))$, involving Riemann-Liouville fractional integrals. Purohit and Raina $[22,29]$ added one more dimension to this study by introducing certain new integral inequalities for synchronous functions, involving the Saigo fractional integral operators [30]. Further, Baleanu et al. [24] established certain generalized integral inequalities for synchronous functions that are related to the Chebyshev functional (1) using the fractional hypergeometric operator, introduced by Curiel and Galué [31].

In this paper, we establish certain integral inequalities related to the weighted Chebyshev's functional (3) in the case of differentiable functions whose derivatives belong to the space $L_{p}([0, \infty))$, involving fractional hypergeometric operators due to [31]. Later, we develop some integral inequalities for the fractional integrals by suitably choosing the function $p(t)$. Some of the known results due to Dahmani et al. [28] and Purohit and Raina [29] follow as special cases of our findings.

Firstly, we give some necessary definitions and mathematical preliminaries of fractional calculus operators which are used further in this paper.

Definition 1. A real-valued function $f(t)(t>0)$ is said to be in the space $\mathbb{C}_{\mu}(\mu \in \mathbb{R})$, if there exists a real number $p>\mu$ such that $f(t)=t^{p} \phi(t)$, where $\phi(t) \in \mathbb{C}(0, \infty)$.

Definition 2. Let $\alpha>0, \mu>-1, \beta, \eta \in \mathbb{R}$; then, a generalized fractional integral $I_{t}^{\alpha, \beta, \eta, \mu}$ (in terms of the
Gauss hypergeometric function) of order $\alpha$ for a real-valued continuous function $f(t)$ is defined by [31] (see also [32])

$$
\begin{aligned}
I_{t}^{\alpha, \beta, \eta, \mu}\{f(t)\}= & \frac{t^{-\alpha-\beta-2 \mu}}{\Gamma(\alpha)} \\
& \quad \times \int_{0}^{t} \tau^{\mu}(t-\tau)^{\alpha-1} \\
& \quad \times{ }_{2} F_{1}\left(\alpha+\beta+\mu,-\eta ; \alpha ; 1-\frac{\tau}{t}\right) f(\tau) d \tau,
\end{aligned}
$$

where the function ${ }_{2} F_{1}(-)$ appearing as a kernel for the operator (5) is the Gaussian hypergeometric function defined by

$$
{ }_{2} F_{1}(a, b ; c ; t)=\sum_{n=0}^{\infty} \frac{(a)_{n}(b)_{n}}{(c)_{n}} \frac{t^{n}}{n !}
$$

and $(a)_{n}$ is the Pochhammer symbol

$$
(a)_{n}=a(a+1) \cdots(a+n-1), \quad(a)_{0}=1 .
$$

It may be noted that the Pochhammer symbol in terms of the gamma function is defined by

$$
(a)_{n}=\frac{\Gamma(a+n)}{\Gamma(a)}, \quad(n>0)
$$

where the gamma function [33] is given by

$$
\Gamma(x)=\int_{0}^{\infty} t^{x-1} e^{-t} d t, \quad(\Re(x)>0) .
$$

Our results in this paper are based on the following preliminary assertions giving composition formula of fractional integral (5) with a power function.

Lemma 3 (see [24]). Let $\alpha, \beta, \eta, \lambda \in \mathbb{R}, \mu>-1, \mu+\lambda>0$, and $\lambda-\beta+\eta>0$; then, the following image formula for the power function under the operator (5) holds true:

$$
I_{t}^{\alpha, \beta, \eta, \mu}\left\{t^{\lambda-1}\right\}=\frac{\Gamma(\mu+\lambda) \Gamma(\lambda-\beta+\eta)}{\Gamma(\lambda-\beta) \Gamma(\lambda+\mu+\alpha+\eta)} t^{\lambda-\beta-\mu-1} .
$$

\section{Main Results}

In this section, we obtain certain integral inequality which gives estimation for the fractional integral of a product in terms of the product of the individual function fractional integrals, involving fractional hypergeometric operators. We give our results related to Chebyshev's functional (3) in the case of differentiable mappings whose derivatives belong to the space $L_{p}([0, \infty))$ satisfying Holder's inequality.

Theorem 4. Let $p$ be a positive function and let $f$ and $g$ be two synchronous functions on $[0, \infty)$. If $f^{\prime} \in L_{r}([0, \infty))$, 
$g^{\prime} \in L_{s}([0, \infty)), r>1, r^{-1}+s^{-1}=1$, then (for all $t>0$, $\beta<1, \mu>-1, \alpha>\max \{0,-\beta-\mu\}, \beta-1<\eta<0)$

$$
\begin{gathered}
2 \mid I_{t}^{\alpha, \beta, \eta, \mu}\{p(t)\} I_{t}^{\alpha, \beta, \eta, \mu}\{p(t) f(t) g(t)\} \\
-I_{t}^{\alpha, \beta, \eta, \mu}\{p(t) f(t)\} I_{t}^{\alpha, \beta, \eta, \mu}\{p(t) g(t)\} \mid \\
\leq \frac{t^{-2 \alpha-2 \beta-4 \mu}\left\|f^{\prime}\right\|_{r}\left\|g^{\prime}\right\|_{s}}{\Gamma^{2}(\alpha)} \\
\quad \times \iint_{0}^{t} \tau^{\mu} \rho^{\mu}(t-\tau)^{\alpha-1}(t-\rho)^{\alpha-1} \\
\quad \times{ }_{2} F_{1}\left(\alpha+\beta+\mu,-\eta ; \alpha ; 1-\frac{\tau}{t}\right) \\
\quad \times{ }_{2} F_{1}\left(\alpha+\beta+\mu,-\eta ; \alpha ; 1-\frac{\rho}{t}\right) \\
\quad \times p(\tau) p(\rho)|\tau-\rho| d \tau d \rho \\
\leq\left\|f^{\prime}\right\|_{r}\left\|g^{\prime}\right\|_{s} t\left(I_{t}^{\alpha, \beta, \eta, \mu}\{p(t)\}\right)^{2} .
\end{gathered}
$$

Proof. Let $f$ and $g$ be two synchronous functions; then, using Definition 1 , for all $\tau, \rho \in(0, t), t \geq 0$, we define

$$
\mathscr{H}(\tau, \rho)=(f(\tau)-f(\rho))(g(\tau)-g(\rho))
$$

Consider

$$
\begin{aligned}
F(t, \tau)= & \frac{t^{-\alpha-\beta-2 \mu} \tau^{\mu}(t-\tau)^{\alpha-1}}{\Gamma(\alpha)} \\
& \times{ }_{2} F_{1}\left(\alpha+\beta+\mu,-\eta ; \alpha ; 1-\frac{\tau}{t}\right) \\
\quad(\tau \in(0, t) ; t>0) & \\
= & \frac{\tau^{\mu}}{\Gamma(\alpha)} \frac{(t-\tau)^{\alpha-1}}{t^{\alpha+\beta+2 \mu}}+\frac{\tau^{\mu}(\alpha+\beta+\mu)(-\eta)}{\Gamma(\alpha+1)} \frac{(t-\tau)^{\alpha}}{t^{\alpha+\beta+2 \mu+1}} \\
& +\frac{\tau^{\mu}(\alpha+\beta+\mu)(\alpha+\beta+\mu+1)(-\eta)(-\eta+1)}{2 \Gamma(\alpha+2)} \\
& \times \frac{(t-\tau)^{\alpha+1}}{t^{\alpha+\beta+2 \mu+2}}+\cdots
\end{aligned}
$$

We observe that each term of the above series is positive in view of the conditions stated with Theorem 4 , and, hence, the function $F(t, \tau)$ remains positive, for all $\tau \in(0, t)(t>0)$.
Multiplying both sides of (12) by $F(t, \tau) p(\tau)$ (where $F(t, \tau)$ is given by (13)) and integrating with respect to $\tau$ from 0 to $t$ and using (5), we get

$$
\begin{aligned}
& \frac{t^{-\alpha-\beta-2 \mu}}{\Gamma(\alpha)} \\
& \quad \times \int_{0}^{t} \tau^{\mu}(t-\tau)^{\alpha-1} \\
& \quad \times{ }_{2} F_{1}\left(\alpha+\beta+\mu,-\eta ; \alpha ; 1-\frac{\tau}{t}\right) p(\tau) \mathscr{H}(\tau, \rho) d \tau \\
& =I_{t}^{\alpha, \beta, \eta, \mu}\{p(t) f(t) g(t)\} \\
& \quad-f(\rho) I_{t}^{\alpha, \beta, \eta, \mu}\{p(t) g(t)\} \\
& \quad-g(\rho) I_{t}^{\alpha, \beta, \eta, \mu}\{p(t) f(t)\}+f(\rho) g(\rho) I_{t}^{\alpha, \beta, \eta, \mu}\{p(t)\} .
\end{aligned}
$$

Next, on multiplying both sides of (14) by $F(t, \rho) p(\rho)$, where $F(t, \rho)$ is given by (13), and integrating with respect to $\rho$ from 0 to $t$, we can write

$$
\begin{aligned}
& \frac{t^{-2 \alpha-2 \beta-4 \mu}}{\Gamma^{2}(\alpha)} \\
& \times \iint_{0}^{t} \tau^{\mu} \rho^{\mu}(t-\tau)^{\alpha-1}(t-\rho)^{\alpha-1} \\
& \quad \times{ }_{2} F_{1}\left(\alpha+\beta+\mu,-\eta ; \alpha ; 1-\frac{\tau}{t}\right) \\
& \times{ }_{2} F_{1}\left(\alpha+\beta+\mu,-\eta ; \alpha ; 1-\frac{\rho}{t}\right) \\
& \times p(\tau) p(\rho) \mathscr{H}(\tau, \rho) d \tau d \rho \\
& =2\left(I_{t}^{\alpha, \beta, \eta, \mu}\{p(t)\} I_{t}^{\alpha, \beta, \eta, \mu}\{p(t) f(t) g(t)\}\right. \\
& \left.-I_{t}^{\alpha, \beta, \eta, \mu}\{p(t) f(t)\} I_{t}^{\alpha, \beta, \eta, \mu}\{p(t) g(t)\}\right) .
\end{aligned}
$$

In view of (12), we have

$$
\mathscr{H}(\tau, \rho)=\iint_{\tau}^{\rho} f^{\prime}(y) g^{\prime}(z) d y d z
$$

Using the following Holder inequality for the double integral:

$$
\begin{array}{r}
\left|\iint_{\tau}^{\rho} f(y) g(z) d y d z\right| \\
\leq\left.\left.\left.\left.\left|\iint_{\tau}^{\rho}\right| f(y)\right|^{r} d y d z\right|^{r^{-1}}\left|\iint_{\tau}^{\rho}\right| g(z)\right|^{s} d y d z\right|^{s^{-1}} \\
\left(r^{-1}+s^{-1}=1\right),
\end{array}
$$

we obtain

$$
|\mathscr{H}(\tau, \rho)| \leq\left.\left.\left.\left.\left|\iint_{\tau}^{\rho}\right| f^{\prime}(y)\right|^{r} d y d z\right|^{r^{-1}}\left|\iint_{\tau}^{\rho}\right| g^{\prime}(z)\right|^{s} d y d z\right|^{s^{-1}} .
$$


Since

$$
\begin{gathered}
\left.\left.\left|\iint_{\tau}^{\rho}\right| f^{\prime}(y)\right|^{r} d y d z\right|^{r^{-1}}=\left.\left.|\tau-\rho|^{r^{-1}}\left|\int_{\tau}^{\rho}\right| f^{\prime}(y)\right|^{r} d y\right|^{r^{-1}}, \\
\left.\left.\left|\iint_{\tau}^{\rho}\right| g^{\prime}(z)\right|^{s} d y d z\right|^{s^{-1}}=\left.\left.|\tau-\rho|^{s^{-1}}\left|\int_{\tau}^{\rho}\right| g^{\prime}(z)\right|^{s} d z\right|^{s^{-1}},
\end{gathered}
$$

then (18) reduces to

$$
|\mathscr{H}(\tau, \rho)| \leq\left.\left.\left.\left.|\tau-\rho|\left|\int_{\tau}^{\rho}\right| f^{\prime}(y)\right|^{r} d y\right|^{r^{-1}}\left|\int_{\tau}^{\rho}\right| g^{\prime}(z)\right|^{s} d z\right|^{s^{-1}} .
$$

It follows from (15) that

$$
\begin{aligned}
& \frac{t^{-2 \alpha-2 \beta-4 \mu}}{\Gamma^{2}(\alpha)} \\
& \quad \times \iint_{0}^{t} \tau^{\mu} \rho^{\mu}(t-\tau)^{\alpha-1}(t-\rho)^{\alpha-1} \\
& \quad \times{ }_{2} F_{1}\left(\alpha+\beta+\mu,-\eta ; \alpha ; 1-\frac{\tau}{t}\right) \\
& \quad \times{ }_{2} F_{1}\left(\alpha+\beta+\mu,-\eta ; \alpha ; 1-\frac{\rho}{t}\right) \\
& \quad \times p(\tau) p(\rho)|\mathscr{H}(\tau, \rho)| d \tau d \rho \\
& \leq \frac{t^{-2 \alpha-2 \beta-4 \mu}}{\Gamma^{2}(\alpha)} \\
& \quad \times \iint_{0}^{t} \tau^{\mu} \rho^{\mu}(t-\tau)^{\alpha-1}(t-\rho)^{\alpha-1} \\
& \quad \times{ }_{2} F_{1}\left(\alpha+\beta+\mu,-\eta ; \alpha ; 1-\frac{\tau}{t}\right) \\
& \quad \times{ }_{2} F_{1}\left(\alpha+\beta+\mu,-\eta ; \alpha ; 1-\frac{\rho}{t}\right) p(\tau) p(\rho) \\
& \quad \times\left.\left.\left.\left.|\tau-\rho|\left|\int_{\tau}^{\rho}\right| f^{\prime}(y)\right|^{r} d y\right|^{r^{-1}}\left|\int_{\tau}^{\rho}\right| g^{\prime}(z)\right|^{s} d z\right|^{s^{-1}} d \tau d \rho
\end{aligned}
$$

Applying again Holder's inequality (17) on the right-hand side of (21), we get

$$
\begin{aligned}
& \frac{t^{-2 \alpha-2 \beta-4 \mu}}{\Gamma^{2}(\alpha)} \\
& \quad \times \iint_{0}^{t} \tau^{\mu} \rho^{\mu}(t-\tau)^{\alpha-1}(t-\rho)^{\alpha-1} \\
& \quad \times{ }_{2} F_{1}\left(\alpha+\beta+\mu,-\eta ; \alpha ; 1-\frac{\tau}{t}\right) \\
& \quad \times{ }_{2} F_{1}\left(\alpha+\beta+\mu,-\eta ; \alpha ; 1-\frac{\rho}{t}\right) \\
& \quad \times p(\tau) p(\rho)|\mathscr{H}(\tau, \rho)| d \tau d \rho
\end{aligned}
$$

$$
\begin{aligned}
& \leq\left[\frac{t^{-r \alpha-r \beta-2 r \mu}}{\Gamma^{r}(\alpha)}\right. \\
& \quad \times \iint_{0}^{t} \tau^{\mu} \rho^{\mu}(t-\tau)^{\alpha-1}(t-\rho)^{\alpha-1} \\
& \quad \times{ }_{2} F_{1}\left(\alpha+\beta+\mu,-\eta ; \alpha ; 1-\frac{\tau}{t}\right) \\
& \quad \times{ }_{2} F_{1}\left(\alpha+\beta+\mu,-\eta ; \alpha ; 1-\frac{\rho}{t}\right) p(\tau) p(\rho) \\
& \left.\quad \times\left.|\tau-\rho|\left|\int_{\tau}^{\rho}\right| f^{\prime}(y)\right|^{r} d y \mid d \tau d \rho\right]^{r^{-1}}
\end{aligned}
$$$$
\times\left[\frac{t^{-s \alpha-s \beta-2 s \mu}}{\Gamma^{s}(\alpha)}\right.
$$$$
\times \iint_{0}^{t} \tau^{\mu} \rho^{\mu}(t-\tau)^{\alpha-1}(t-\rho)^{\alpha-1}
$$$$
\times{ }_{2} F_{1}\left(\alpha+\beta+\mu,-\eta ; \alpha ; 1-\frac{\tau}{t}\right)
$$$$
\times{ }_{2} F_{1}\left(\alpha+\beta+\mu,-\eta ; \alpha ; 1-\frac{\rho}{t}\right) p(\tau) p(\rho)
$$$$
\left.\times\left.|\tau-\rho|\left|\int_{\tau}^{\rho}\right| g^{\prime}(z)\right|^{s} d z \mid d \tau d \rho\right]^{s^{-1}} .
$$

In view of the fact that

$$
\left.\left|\int_{\tau}^{\rho}\right| f(y)\right|^{p} d y \mid \leq\|f\|_{p}^{p},
$$

we get

$$
\begin{gathered}
\frac{t^{-2 \alpha-2 \beta-4 \mu}}{\Gamma^{2}(\alpha)} \\
\quad \times \iint_{0}^{t} \tau^{\mu} \rho^{\mu}(t-\tau)^{\alpha-1}(t-\rho)^{\alpha-1} \\
\quad \times{ }_{2} F_{1}\left(\alpha+\beta+\mu,-\eta ; \alpha ; 1-\frac{\tau}{t}\right) \\
\quad \times{ }_{2} F_{1}\left(\alpha+\beta+\mu,-\eta ; \alpha ; 1-\frac{\rho}{t}\right) \\
\quad \times p(\tau) p(\rho)|\mathscr{H}(\tau, \rho)| d \tau d \rho \\
\leq \quad \frac{t^{-r \alpha-r \beta-2 r \mu}\left\|f^{\prime}\right\|_{r}^{r}}{\Gamma^{r}(\alpha)} \\
\quad \times \iint_{0}^{t} \tau^{\mu} \rho^{\mu}(t-\tau)^{\alpha-1}(t-\rho)^{\alpha-1}
\end{gathered}
$$




$$
\begin{aligned}
& \times{ }_{2} F_{1}\left(\alpha+\beta+\mu,-\eta ; \alpha ; 1-\frac{\tau}{t}\right) \\
& \times{ }_{2} F_{1}\left(\alpha+\beta+\mu,-\eta ; \alpha ; 1-\frac{\rho}{t}\right) \\
& \times p(\tau) p(\rho)|\tau-\rho| d \tau d \rho]^{r^{-1}} \\
& \times\left[\frac{t^{-s \alpha-s \beta-2 s \mu}\left\|g^{\prime}\right\|_{s}^{s}}{\Gamma^{s}(\alpha)}\right. \\
& \times \iint_{0}^{t} \tau^{\mu} \rho^{\mu}(t-\tau)^{\alpha-1}(t-\rho)^{\alpha-1} \\
& \times{ }_{2} F_{1}\left(\alpha+\beta+\mu,-\eta ; \alpha ; 1-\frac{\tau}{t}\right) \\
& \times{ }_{2} F_{1}\left(\alpha+\beta+\mu,-\eta ; \alpha ; 1-\frac{\rho}{t}\right) \\
& \times p(\tau) p(\rho)|\tau-\rho| d \tau d \rho]^{s^{-1}}
\end{aligned}
$$

From (24), we obtain

$$
\begin{aligned}
& \frac{t^{-2 \alpha-2 \beta-4 \mu}}{\Gamma^{2}(\alpha)} \\
& \times \iint_{0}^{t} \tau^{\mu} \rho^{\mu}(t-\tau)^{\alpha-1}(t-\rho)^{\alpha-1} \\
& \times{ }_{2} F_{1}\left(\alpha+\beta+\mu,-\eta ; \alpha ; 1-\frac{\tau}{t}\right) \\
& \times{ }_{2} F_{1}\left(\alpha+\beta+\mu,-\eta ; \alpha ; 1-\frac{\rho}{t}\right) \\
& \times p(\tau) p(\rho)|\mathscr{H}(\tau, \rho)| d \tau d \rho \\
& \leq \frac{t^{-2 \alpha-2 \beta-4 \mu}\left\|f^{\prime}\right\|_{r}\left\|g^{\prime}\right\|_{s}}{\Gamma^{2}(\alpha)} \\
& \times\left[\iint_{0}^{t} \tau^{\mu} \rho^{\mu}(t-\tau)^{\alpha-1}(t-\rho)^{\alpha-1}\right. \\
& \times{ }_{2} F_{1}\left(\alpha+\beta+\mu,-\eta ; \alpha ; 1-\frac{\tau}{t}\right) \\
& \times{ }_{2} F_{1}\left(\alpha+\beta+\mu,-\eta ; \alpha ; 1-\frac{\rho}{t}\right) \\
& \times p(\tau) p(\rho)|\tau-\rho| d \tau d \rho]^{r^{-1}} \\
& \times\left[\iint_{0}^{t} \tau^{\mu} \rho^{\mu}(t-\tau)^{\alpha-1}(t-\rho)^{\alpha-1}\right. \\
& \times{ }_{2} F_{1}\left(\alpha+\beta+\mu,-\eta ; \alpha ; 1-\frac{\tau}{t}\right)
\end{aligned}
$$

$$
\begin{aligned}
& \times{ }_{2} F_{1}\left(\alpha+\beta+\mu,-\eta ; \alpha ; 1-\frac{\rho}{t}\right) \\
& \times p(\tau) p(\rho)|\tau-\rho| d \tau d \rho]^{s^{-1}} .
\end{aligned}
$$

Since $r^{-1}+s^{-1}=1$, the above inequality yields

$$
\begin{aligned}
& \frac{t^{-2 \alpha-2 \beta-4 \mu}}{\Gamma^{2}(\alpha)} \\
& \times \iint_{0}^{t} \tau^{\mu} \rho^{\mu}(t-\tau)^{\alpha-1}(t-\rho)^{\alpha-1} \\
& \quad \times{ }_{2} F_{1}\left(\alpha+\beta+\mu,-\eta ; \alpha ; 1-\frac{\tau}{t}\right) \\
& \quad \times{ }_{2} F_{1}\left(\alpha+\beta+\mu,-\eta ; \alpha ; 1-\frac{\rho}{t}\right) \\
& \quad \times p(\tau) p(\rho)|\mathscr{H}(\tau, \rho)| d \tau d \rho \\
& \leq \frac{t^{-2 \alpha-2 \beta-4 \mu}\left\|f^{\prime}\right\|\left\|_{r}\right\| g_{s}}{\Gamma^{2}(\alpha)} \\
& \times \iint_{0}^{t} \tau^{\mu} \rho^{\mu}(t-\tau)^{\alpha-1}(t-\rho)^{\alpha-1} \\
& \quad \times{ }_{2} F_{1}\left(\alpha+\beta+\mu,-\eta ; \alpha ; 1-\frac{\tau}{t}\right) \\
& \quad \times{ }_{2} F_{1}\left(\alpha+\beta+\mu,-\eta ; \alpha ; 1-\frac{\rho}{t}\right) \\
& \times p^{\prime} p(\tau) p(\rho)|\tau-\rho| d \tau d \rho,
\end{aligned}
$$

which in view of (15) gives

$$
\begin{aligned}
& 2 \mid I_{t}^{\alpha, \beta, \eta, \mu}\{p(t)\} I_{t}^{\alpha, \beta, \eta, \mu}\{p(t) f(t) g(t)\} \\
& -I_{t}^{\alpha, \beta, \eta, \mu}\{p(t) f(t)\} I_{t}^{\alpha, \beta, \eta, \mu}\{p(t) g(t)\} \mid \\
& \leq \frac{t^{-2 \alpha-2 \beta-4 \mu}}{\Gamma^{2}(\alpha)} \\
& \quad \times \iint_{0}^{t} \tau^{\mu} \rho^{\mu}(t-\tau)^{\alpha-1}(t-\rho)^{\alpha-1} \\
& \quad \times{ }_{2} F_{1}\left(\alpha+\beta+\mu,-\eta ; \alpha ; 1-\frac{\tau}{t}\right) \\
& \quad \times{ }_{2} F_{1}\left(\alpha+\beta+\mu,-\eta ; \alpha ; 1-\frac{\rho}{t}\right) \\
& \quad \times p(\tau) p(\rho)|\mathscr{H}(\tau, \rho)| d \tau d \rho .
\end{aligned}
$$

Making use of (26) and (27), the left-hand side of the inequality (11) follows.

To prove the right-hand side of the inequality (11), we observe that $0 \leq \tau \leq t, 0 \leq \rho \leq t$, and, therefore,

$$
0 \leq|\tau-\rho| \leq t \text {. }
$$


Evidently from (26), we get

$$
\begin{aligned}
& \frac{t^{-2 \alpha-2 \beta-4 \mu}}{\Gamma^{2}(\alpha)} \\
& \quad \times \iint_{0}^{t} \tau^{\mu} \rho^{\mu}(t-\tau)^{\alpha-1}(t-\rho)^{\alpha-1} \\
& \quad \times{ }_{2} F_{1}\left(\alpha+\beta+\mu,-\eta ; \alpha ; 1-\frac{\tau}{t}\right) \\
& \quad \times{ }_{2} F_{1}\left(\alpha+\beta+\mu,-\eta ; \alpha ; 1-\frac{\rho}{t}\right) \\
& \quad \times p(\tau) p(\rho)|\mathscr{H}(\tau, \rho)| d \tau d \rho \\
& \quad \frac{t^{-2 \alpha-2 \beta-4 \mu}\left\|f^{\prime}\right\|{ }_{r}\|\|_{s} t}{\Gamma^{2}(\alpha)} \\
& \quad \times \iint_{0}^{t} \tau^{\mu} \rho^{\mu}(t-\tau)^{\alpha-1}(t-\rho)^{\alpha-1} \\
& \quad \times{ }_{2} F_{1}\left(\alpha+\beta+\mu,-\eta ; \alpha ; 1-\frac{\tau}{t}\right) \\
& \quad \times{ }_{2} F_{1}\left(\alpha+\beta+\mu,-\eta ; \alpha ; 1-\frac{\rho}{t}\right) \\
& \times f_{r}\left\|_{r}\right\| g^{\prime} \|_{s} t\left(I_{t}^{\alpha, \beta, \eta, \mu}\{p(t)\}\right)^{2},
\end{aligned}
$$

which completes the proof of Theorem 4.

The following gives a generalization of Theorem 4 .

Theorem 5. Let $p$ be a positive function and let $f$ and $g$ be two synchronous functions on $[0, \infty)$. If $f^{\prime} \in L_{r}([0, \infty)), g^{\prime} \in$ $L_{s}([0, \infty)), r>1, r^{-1}+s^{-1}=1$, then

$$
\begin{gathered}
\mid I_{t}^{\alpha, \beta, \eta, \mu}\{p(t)\} I_{t}^{\gamma, \delta, \zeta, \nu}\{p(t) f(t) g(t)\} \\
+I_{t}^{\gamma, \delta, \zeta, v}\{p(t)\} I_{t}^{\alpha, \beta, \eta, \mu}\{p(t) f(t) g(t)\} \\
-I_{t}^{\alpha, \beta, \eta, \mu}\{p(t) f(t)\} I_{t}^{\gamma, \delta, \zeta, v}\{p(t) g(t)\} \\
-I_{t}^{\gamma, \delta, \zeta, \nu}\{p(t) f(t)\} I_{t}^{\alpha, \beta, \eta, \mu}\{p(t) g(t)\} \mid \\
\leq \frac{t^{-\alpha-\beta-\gamma-\delta-2(\mu+\nu)}\left\|f^{\prime}\right\|_{r}\left\|g^{\prime}\right\|_{s}}{\Gamma(\alpha) \Gamma(\gamma)} \\
\times \iint_{0}^{t} \tau^{\mu} \rho^{\nu}(t-\tau)^{\alpha-1}(t-\rho)^{\gamma-1} \\
\times{ }_{2} F_{1}\left(\alpha+\beta+\mu,-\eta ; \alpha ; 1-\frac{\tau}{t}\right) \\
\quad \times{ }_{2} F_{1}\left(\gamma+\delta+\nu,-\zeta ; \gamma ; 1-\frac{\rho}{t}\right) \\
\times p p(\tau) p(\rho)|\tau-\rho| d \tau d \rho \\
\leq\left\|f^{\prime}\right\|\left\|_{r}\right\| g^{\prime} \| t I_{t}^{\alpha, \beta, \eta, \mu}\{p(t)\} I_{t}^{\gamma, \delta, \zeta, v}\{p(t)\}
\end{gathered}
$$

for all $t>0, \alpha>\max \{0,-\beta-\mu\}, \beta<1, \mu>-1, \beta-1<\eta<0$, $\gamma>\max \{0,-\delta-\nu\}, \delta<1, \nu>-1, \delta-1<\zeta<0$.

Proof. To prove the above theorem, we use inequality (14). Multiplying both sides of (14) by

$$
\begin{array}{r}
\frac{t^{-\gamma-\delta-2 v} \rho^{\nu}(t-\rho)^{\gamma-1}}{\Gamma(\gamma)}{ }_{2} F_{1}\left(\gamma+\delta+\nu,-\zeta ; \gamma ; 1-\frac{\rho}{t}\right) p(\rho), \\
(\rho \in(0, t) ; t>0),
\end{array}
$$

which remains positive in view of the conditions stated with (30) and then integrating with respect to $\rho$ from 0 to $t$, we get

$$
\begin{gathered}
\frac{t^{-\alpha-\beta-\gamma-\delta-2(\mu+\nu)}}{\Gamma(\alpha) \Gamma(\gamma)} \\
\times \iint_{0}^{t}(t-\tau)^{\alpha-1}(t-\rho)^{\gamma-1} \\
\times{ }_{2} F_{1}\left(\alpha+\beta+\mu,-\eta ; \alpha ; 1-\frac{\tau}{t}\right) \\
\times{ }_{2} F_{1}\left(\gamma+\delta+\nu,-\zeta ; \gamma ; 1-\frac{\rho}{t}\right) \\
\times \tau^{\mu} \rho^{\nu} p(\tau) p(\rho) \mathscr{H}(\tau, \rho) d \tau d \rho \\
=I_{t}^{\alpha, \beta, \eta, \mu}\{p(t)\} I_{t}^{\gamma, \delta, \zeta, v}\{p(t) f(t) g(t)\} \\
+I_{t}^{\gamma, \delta, \zeta, \nu}\{p(t)\} I_{t}^{\alpha, \beta, \eta, \mu}\{p(t) f(t) g(t)\} \\
-I_{t}^{\alpha, \beta, \eta, \mu}\{p(t) f(t)\} I_{t}^{\gamma, \delta, \zeta, \nu}\{p(t) g(t)\} \\
-I_{t}^{\gamma, \delta, \zeta, v}\{p(t) f(t)\} I_{t}^{\alpha, \beta, \eta, \mu}\{p(t) g(t)\} .
\end{gathered}
$$

Now making use of (20), then (32) gives

$$
\begin{aligned}
& \frac{t^{-\alpha-\beta-\gamma-\delta-2(\mu+\nu)}}{\Gamma(\alpha) \Gamma(\gamma)} \\
& \quad \times \iint_{0}^{t}(t-\tau)^{\alpha-1}(t-\rho)^{\gamma-1} \\
& \quad \times{ }_{2} F_{1}\left(\alpha+\beta+\mu,-\eta ; \alpha ; 1-\frac{\tau}{t}\right) \\
& \quad \times{ }_{2} F_{1}\left(\gamma+\delta+\nu,-\zeta ; \gamma ; 1-\frac{\rho}{t}\right) \\
& \quad \times \tau^{\mu} \rho^{\nu} p(\tau) p(\rho)|\mathscr{H}(\tau, \rho)| d \tau d \rho
\end{aligned}
$$




$$
\begin{aligned}
& \leq \frac{t^{-\alpha-\beta-\gamma-\delta-2(\mu+\nu)}}{\Gamma(\alpha) \Gamma(\gamma)} \\
& \times \iint_{0}^{t} \tau^{\mu} \rho^{\nu}(t-\tau)^{\alpha-1}(t-\rho)^{\gamma-1} \\
& \quad \times{ }_{2} F_{1}\left(\alpha+\beta+\mu,-\eta ; \alpha ; 1-\frac{\tau}{t}\right) \\
& \quad \times{ }_{2} F_{1}\left(\gamma+\delta+\nu,-\zeta ; \gamma ; 1-\frac{\rho}{t}\right) p(\tau) p(\rho) \\
& \quad \times\left.\left.|\tau-\rho|\left|\int_{\tau}^{\rho}\right| f^{\prime}(y)\right|^{r} d y\right|^{r^{-1}} \\
& \quad \times\left.\left.\left|\int_{\tau}^{\rho}\right| g^{\prime}(z)\right|^{s} d z\right|^{s^{-1}} d \tau d \rho .
\end{aligned}
$$

Applying Holder's inequality (17) on the right-hand side of (33), we get

$$
\begin{aligned}
& \frac{t^{-\alpha-\beta-\gamma-\delta-2(\mu+\nu)}}{\Gamma(\alpha) \Gamma(\gamma)} \\
& \times \iint_{0}^{t}(t-\tau)^{\alpha-1}(t-\rho)^{\gamma-1} \\
& \quad \times{ }_{2} F_{1}\left(\alpha+\beta+\mu,-\eta ; \alpha ; 1-\frac{\tau}{t}\right) \\
& \quad \times{ }_{2} F_{1}\left(\gamma+\delta+\nu,-\zeta ; \gamma ; 1-\frac{\rho}{t}\right) \\
& \quad \times \frac{\tau^{\mu} \rho^{\nu} p(\tau) p(\rho)|\mathscr{H}(\tau, \rho)| d \tau d \rho}{\Gamma^{-r \alpha-r \beta-2 r \mu}(\alpha)} \\
& \quad \times \iint_{0}^{t} \tau^{\mu} \rho^{\nu}(t-\tau)^{\alpha-1}(t-\rho)^{\gamma-1} \\
& \quad \times\left[{ }_{2} F_{1}\left(\alpha+\beta+\mu,-\eta ; \alpha ; 1-\frac{\tau}{t}\right)\right. \\
& \quad \times{ }_{2} F_{1}{ }^{-s \gamma-s \delta-2 s \nu}\left(\gamma+\delta+\nu,-\zeta ; \gamma ; 1-\frac{\rho}{t}\right) p(\tau) p(\rho) \\
& \left.\quad \times\left.|\tau-\rho|\left|\int_{\tau} \tau^{\mu}\right| f^{\prime}(y)\right|^{r} d y \mid d \tau d \rho\right]^{r^{-1}}(t-\tau)^{\alpha-1}(t-\rho)^{\gamma-1}
\end{aligned}
$$

$$
\begin{aligned}
& \times{ }_{2} F_{1}\left(\alpha+\beta+\mu,-\eta ; \alpha ; 1-\frac{\tau}{t}\right) \\
& \times{ }_{2} F_{1}\left(\gamma+\delta+\mu,-\zeta ; \gamma ; 1-\frac{\rho}{t}\right) p(\tau) p(\rho) \\
& \left.\times\left.|\tau-\rho|\left|\int_{\tau}^{\rho}\right| g^{\prime}(z)\right|^{s} d z \mid d \tau d \rho\right]^{s^{-1}},
\end{aligned}
$$

which on using (23) readily yields the following inequality:

$$
\begin{aligned}
& \frac{t^{-\alpha-\beta-\gamma-\delta-2(\mu+\nu)}}{\Gamma(\alpha) \Gamma(\gamma)} \\
& \times \iint_{0}^{t}(t-\tau)^{\alpha-1}(t-\rho)^{\gamma-1} \\
& \quad \times{ }_{2} F_{1}\left(\alpha+\beta+\mu,-\eta ; \alpha ; 1-\frac{\tau}{t}\right) \\
& \quad \times{ }_{2} F_{1}\left(\gamma+\delta+\nu,-\zeta ; \gamma ; 1-\frac{\rho}{t}\right) \\
& \quad \times \tau^{\mu} \rho^{\nu} p(\tau) p(\rho)|\mathscr{H}(\tau, \rho)| d \tau d \rho \\
& \leq \frac{t^{-\alpha-\beta-\gamma-\delta-2(\mu+\nu)}\left\|f^{\prime}\right\|_{r}\left\|g^{\prime}\right\|_{s}}{\Gamma(\alpha) \Gamma(\gamma)} \\
& \times \iint_{0}^{t} \tau^{\mu} \rho^{\nu}(t-\tau)^{\alpha-1}(t-\rho)^{\gamma-1} \\
& \quad \times{ }_{2} F_{1}\left(\alpha+\beta+\mu,-\eta ; \alpha ; 1-\frac{\tau}{t}\right) \\
& \quad \times{ }_{2} F_{1}\left(\gamma+\delta+\nu,-\zeta ; \gamma ; 1-\frac{\rho}{t}\right) \\
& \quad \times p(\tau) p(\rho)|\tau-\rho| d \tau d \rho .
\end{aligned}
$$

In view of (32) and (35) and the properties of modulus, one can easily arrive at the left-sided inequality of Theorem 5 . Moreover, we have $0 \leq \tau \leq t, 0 \leq \rho \leq t$; hence,

$$
0 \leq|\tau-\rho| \leq t
$$

Therefore, from (35), we get

$$
\begin{aligned}
& \frac{t^{-\alpha-\beta-\gamma-\delta-2(\mu+\gamma)}}{\Gamma(\alpha) \Gamma(\gamma)} \\
& \quad \times \iint_{0}^{t}(t-\tau)^{\alpha-1}(t-\rho)^{\gamma-1} \\
& \quad \times{ }_{2} F_{1}\left(\alpha+\beta+\mu,-\eta ; \alpha ; 1-\frac{\tau}{t}\right) \\
& \quad \times{ }_{2} F_{1}\left(\gamma+\delta+\nu,-\zeta ; \gamma ; 1-\frac{\rho}{t}\right)
\end{aligned}
$$




$$
\begin{gathered}
\times \tau^{\mu} \rho^{\nu} p(\tau) p(\rho)|\mathscr{H}(\tau, \rho)| d \tau d \rho \\
\leq \frac{t^{-\alpha-\beta-\gamma-\delta-2(\mu+\nu)}\left\|f^{\prime}\right\|_{r}\left\|g^{\prime}\right\|_{s} t}{\Gamma(\alpha) \Gamma(\gamma)} \\
\times \iint_{0}^{t} \tau^{\mu} \rho^{\nu}(t-\tau)^{\alpha-1}(t-\rho)^{\gamma-1} \\
\times{ }_{2} F_{1}\left(\alpha+\beta+\mu,-\eta ; \alpha ; 1-\frac{\tau}{t}\right) \\
\times{ }_{2} F_{1}\left(\gamma+\delta+\nu,-\zeta ; \gamma ; 1-\frac{\rho}{t}\right) p(\tau) p(\rho) d \tau d \rho \\
\leq\left\|f^{\prime}\right\|_{r}\left\|g^{\prime}\right\|_{s} I_{t}^{\alpha, \beta, \eta, \mu}\{p(t)\} I_{t}^{\gamma, \delta, \zeta, \nu}\{p(t)\},
\end{gathered}
$$

which completes the proof of Theorem 5 .

Remark 6. For $\gamma=\alpha, \delta=\beta, \zeta=\eta$, and $\nu=\mu$, Theorem 5 immediately reduces to Theorem 4 .

\section{Consequent Results and Special Cases}

As implications of our main results, we consider some consequent results of Theorems 4 and 5 by suitably choosing the function $p(t)$. To this end, let us set $p(t)=t^{\lambda}(\lambda \in$ $[0, \infty), t \in(0, \infty))$; then, on using (10), Theorems 4 and 5 yield the following results.

Corollary 7. Let $f$ and $g$ be two synchronous functions on $[0, \infty)$. If $f^{\prime} \in L_{r}([0, \infty)), g^{\prime} \in L_{s}([0, \infty)), r>1, r^{-1}+s^{-1}=$ 1 , then

$$
\begin{aligned}
& 2 \mid \frac{\Gamma(\mu+\lambda+1) \Gamma(\lambda+1-\beta+\eta)}{\Gamma(\lambda+1-\beta) \Gamma(\lambda+\mu+1+\alpha+\eta)} \\
& \quad \times t^{\lambda-\beta-\mu} I_{t}^{\alpha, \beta, \eta, \mu}\left\{t^{\lambda} f(t) g(t)\right\} \\
& \quad-I_{t}^{\alpha, \beta, \eta, \mu}\left\{t^{\lambda} f(t)\right\} I_{t}^{\alpha, \beta, \eta, \mu}\left\{t^{\lambda} g(t)\right\} \mid \\
& \leq \frac{t^{-2 \alpha-2 \beta-4 \mu}\left\|f^{\prime}\right\|_{r}\left\|g^{\prime}\right\|_{s}}{\Gamma^{2}(\alpha)} \\
& \quad \times \iint_{0}^{t} \tau^{\mu+\lambda} \rho^{\mu+\lambda}(t-\tau)^{\alpha-1}(t-\rho)^{\alpha-1} \\
& \quad \times{ }_{2} F_{1}\left(\alpha+\beta+\mu,-\eta ; \alpha ; 1-\frac{\tau}{t}\right) \\
& \quad \times{ }_{2} F_{1}\left(\alpha+\beta+\mu,-\eta ; \alpha ; 1-\frac{\rho}{t}\right)|\tau-\rho| d \tau d \rho \\
& \leq\left\|f^{\prime}\right\|_{r}\left\|g^{\prime}\right\|_{s} \frac{\Gamma^{2}(\mu+\lambda+1) \Gamma^{2}(\lambda+1-\beta+\eta) t^{1+2 \lambda-2 \beta-2 \mu}}{\Gamma^{2}(\lambda+1-\beta) \Gamma^{2}(\mu+\lambda+1+\alpha+\eta)},
\end{aligned}
$$

wheret $>0, \beta<1, \mu>-1, \alpha>\max \{0,-\beta-\mu\}, \beta-1<\eta<0$, $\lambda \geq 0, \min (\lambda+\mu, \lambda-\beta+\eta)>-1$.
Corollary 8. Let $f$ and $g$ be two synchronous functions on $[0, \infty)$. If $f^{\prime} \in L_{r}([0, \infty)), g^{\prime} \in L_{s}([0, \infty)), r>1, r^{-1}+s^{-1}=$ 1 , then

$$
\begin{aligned}
& \mid \frac{\Gamma(\mu+\lambda+1) \Gamma(\lambda+1-\beta+\eta)}{\Gamma(\lambda+1-\beta) \Gamma(\mu+\lambda+1+\alpha+\eta)} \\
& \times t^{\lambda-\beta-\mu} I_{t}^{\gamma, \delta, \zeta, \nu}\left\{t^{\lambda} f(t) g(t)\right\} \\
& +\frac{\Gamma(\nu+\lambda+1) \Gamma(\lambda+1-\delta+\zeta)}{\Gamma(\lambda+1-\delta) \Gamma(\nu+\lambda+1+\gamma+\zeta)} t^{\lambda-\delta-\nu} \\
& \times I_{t}^{\alpha, \beta, \eta, \mu}\left\{t^{\lambda} f(t) g(t)\right\} \\
& -I_{t}^{\alpha, \beta, \eta, \mu}\left\{t^{\lambda} f(t)\right\} I_{t}^{\gamma, \delta, \zeta, \nu}\left\{t^{\lambda} g(t)\right\} \\
& -I_{t}^{\gamma, \delta, \zeta, \nu}\left\{t^{\lambda} f(t)\right\} I_{t}^{\alpha, \beta, \eta, \mu}\left\{t^{\lambda} g(t)\right\} \\
& \leq \frac{t^{-\alpha-\beta-\gamma-\delta-2 \mu-2 \nu}\left\|f^{\prime}\right\|_{r}\left\|g^{\prime}\right\|_{s}}{\Gamma(\alpha) \Gamma(\gamma)} \\
& \times \iint_{0}^{t}(t-\tau)^{\alpha-1}(t-\rho)^{\gamma-1}{ }_{2} F_{1}\left(\alpha+\beta+\mu,-\eta ; \alpha ; 1-\frac{\tau}{t}\right) \\
& \times{ }_{2} F_{1}\left(\gamma+\delta+\nu,-\zeta ; \gamma ; 1-\frac{\rho}{t}\right) \tau^{\mu+\lambda} \rho^{\nu+\lambda}|\tau-\rho| d \tau d \rho \\
& \leq\left\|f^{\prime}\right\|_{r}\left\|g^{\prime}\right\|_{s} \\
& \times(\Gamma(\mu+\lambda+1) \Gamma(\nu+\lambda+1) \\
& \times \Gamma(\lambda+1-\beta+\eta) \Gamma(\lambda+1-\delta+\zeta)) \\
& \times(\Gamma(\lambda+1-\beta) \Gamma(\mu+\lambda+1+\alpha+\eta) \\
& \times \Gamma(\lambda+1-\delta) \Gamma(\nu+\lambda+1+\gamma+\zeta))^{-1} \\
& \times t^{1+2 \lambda-\beta-\delta-2 \mu-2 v},
\end{aligned}
$$

for all t $>0, \beta<1, \mu>-1, \alpha>\max \{0,-\beta-\mu\}, \beta-1<\eta<0$, $\delta<1, \nu>-1, \gamma>\max \{0,-\delta-\nu\}, \delta-1<\zeta<0, \lambda \geq 0$, $\min (\lambda+\mu, \lambda+\nu, \lambda-\beta+\eta, \lambda-\delta+\zeta)>-1$.

Further, if we put $\lambda=0$ in Corollaries 7 and 8 (or $p(t)=$ 1 in Theorems 4 and 5), we obtain the following integral inequalities.

Corollary 9. Let $f$ and $g$ be two synchronous functions on $[0, \infty)$. If $f^{\prime} \in L_{r}([0, \infty)), g^{\prime} \in L_{s}([0, \infty)), r>1, r^{-1}+s^{-1}=$ 1 , then

$$
\begin{aligned}
& 2 \mid \frac{\Gamma(\mu+1) \Gamma(1-\beta+\eta)}{\Gamma(1-\beta) \Gamma(\mu+1+\alpha+\eta)} t^{-\beta-\mu} I_{t}^{\alpha, \beta, \eta, \mu}\{f(t) g(t)\} \\
& \quad-I_{t}^{\alpha, \beta, \eta, \mu}\{f(t)\} I_{t}^{\alpha, \beta, \eta, \mu}\{g(t)\} \mid
\end{aligned}
$$




$$
\begin{aligned}
& \leq \frac{t^{-2 \alpha-2 \beta-4 \mu}\left\|f^{\prime}\right\|_{r}\left\|g^{\prime}\right\|_{s}}{\Gamma^{2}(\alpha)} \\
& \quad \times \iint_{0}^{t} \tau^{\mu} \rho^{\mu}(t-\tau)^{\alpha-1}(t-\rho)^{\alpha-1} \\
& \quad \times{ }_{2} F_{1}\left(\alpha+\beta+\mu,-\eta ; \alpha ; 1-\frac{\tau}{t}\right) \\
& \quad \times{ }_{2} F_{1}\left(\alpha+\beta+\mu,-\eta ; \alpha ; 1-\frac{\rho}{t}\right)|\tau-\rho| d \tau d \rho \\
& \leq\left\|f^{\prime}\right\|_{r}\left\|g^{\prime}\right\|_{s} \frac{\Gamma^{2}(\mu+1) \Gamma^{2}(1-\beta+\eta) t^{1-2 \beta-2 \mu}}{\Gamma^{2}(1-\beta) \Gamma^{2}(\mu+1+\alpha+\eta)},
\end{aligned}
$$

where $t>0, \beta<1, \mu>-1, \alpha>\max \{0,-\beta-\mu\}, \beta-1<\eta<0$.

Corollary 10. Let $f$ and $g$ be two synchronous functions on $[0, \infty)$. If $f^{\prime} \in L_{r}([0, \infty)), g^{\prime} \in L_{s}([0, \infty)), r>1, r^{-1}+s^{-1}=$ 1 , then

$$
\begin{aligned}
& \mid \frac{\Gamma(\mu+1) \Gamma(1-\beta+\eta)}{\Gamma(1-\beta) \Gamma(\mu+1+\alpha+\eta)} \\
& \times t^{-\beta-\mu} I_{t}^{\gamma, \delta, \zeta, \nu}\{f(t) g(t)\} \\
& +\frac{\Gamma(\nu+1) \Gamma(1-\delta+\zeta)}{\Gamma(1-\delta) \Gamma(\nu+1+\gamma+\zeta)} t^{-\delta-\nu} \\
& \times I_{t}^{\alpha, \beta, \eta, \mu}\{f(t) g(t)\}-I_{t}^{\alpha, \beta, \eta, \mu}\{f(t)\} I_{t}^{\gamma, \delta, \zeta, \nu}\{g(t)\} \\
& -I_{t}^{\gamma, \delta, \zeta, v}\{f(t)\} I_{t}^{\alpha, \beta, \eta, \mu}\{g(t)\} \\
& \leq \frac{t^{-\alpha-\beta-\gamma-\delta-2 \mu-2 \nu}\left\|f^{\prime}\right\|_{r}\left\|g^{\prime}\right\|_{s}}{\Gamma(\alpha) \Gamma(\gamma)} \\
& \times \iint_{0}^{t}(t-\tau)^{\alpha-1}(t-\rho)^{\gamma-1} \\
& \times{ }_{2} F_{1}\left(\alpha+\beta+\mu,-\eta ; \alpha ; 1-\frac{\tau}{t}\right) \\
& \times{ }_{2} F_{1}\left(\gamma+\delta+\nu,-\zeta ; \gamma ; 1-\frac{\rho}{t}\right) \tau^{\mu} \rho^{\nu} \\
& \times|\tau-\rho| d \tau d \rho \\
& \leq\left\|f^{\prime}\right\|_{r}\left\|g^{\prime}\right\|_{s} \\
& \times \frac{\Gamma(\mu+1) \Gamma(\nu+1) \Gamma(1-\beta+\eta) \Gamma(1-\delta+\zeta)}{\Gamma(1-\beta) \Gamma(\mu+1+\alpha+\eta) \Gamma(1-\delta) \Gamma(\nu+1+\gamma+\zeta)} \\
& \times t^{1-\beta-\delta-2 \mu-2 \nu} \text {, }
\end{aligned}
$$

for all $t>0, \beta<1, \mu>-1, \alpha>\max \{0,-\beta-\mu\}, \beta-1<\eta<0$, $\delta<1, \nu>-1, \gamma>\max \{0,-\delta-\nu\}, \delta-1<\zeta<0$.

We now, briefly consider some consequences of the results derived in the previous section. Following Curiel and
Galué [31], the operator (5) would reduce immediately to the extensively investigated Saigo, Erdélyi-Kober, and RiemannLiouville type fractional integral operators, respectively, given by the following relationships (see also $[30,32]$ ):

$$
\begin{aligned}
I_{0, t}^{\alpha, \beta, \eta}\{f(t)\}= & I_{t}^{\alpha, \beta, \eta, 0}\{f(t)\} \\
= & \frac{t^{-\alpha-\beta}}{\Gamma(\alpha)} \int_{0}^{t}(t-\tau)^{\alpha-1} \\
& \quad \times{ }_{2} F_{1}\left(\alpha+\beta,-\eta ; \alpha ; 1-\frac{\tau}{t}\right) f(\tau) d \tau,
\end{aligned}
$$$$
(\alpha>0, \beta, \eta \in \mathbb{R}),
$$

$$
\begin{aligned}
I^{\alpha, \eta}\{f(t)\} & =I_{t}^{\alpha, 0, \eta, 0}\{f(t)\} \\
& =\frac{t^{-\alpha-\eta}}{\Gamma(\alpha)} \int_{0}^{t}(t-\tau)^{\alpha-1} \tau^{\eta} f(\tau) d \tau, \\
& (\alpha>0, \eta \in \mathbb{R}) \\
R^{\alpha}\{f(t)\} & =I_{t}^{\alpha,-\alpha, \eta, 0}\{f(t)\} \\
& =\frac{1}{\Gamma(\alpha)} \int_{0}^{t}(t-\tau)^{\alpha-1} f(\tau) d \tau, \quad(\alpha>0) .
\end{aligned}
$$

Now, if we consider $\mu=0$ (and $\nu=0$ additionally for Theorem 5) and make use of the relation (42), Theorems 4 and 5 provide, respectively, the known fractional integral inequalities due to Purohit and Raina [29]. Again, if we set $\mu=0$ and replace $\beta$ by $-\alpha$ (and $\nu=0$ and replace $\delta$ by $-\gamma$ additionally for Theorem 5) and make use of the relation (44), then Theorems 4 and 5 correspond to the known integral inequalities due to Dahmani et al. [28, pages 39-42, Theorems 3.1 to 3.2 ] involving the Riemann-Liouville type fractional integral operator.

Lastly, we conclude this paper by remarking that we have introduced new general extensions of Chebyshev type inequalities involving fractional integral hypergeometric operators. By suitably specializing the arbitrary function $p(t)$, one can further easily obtain additional integral inequalities involving the Riemann-Liouville, Erdélyi-Kober, and Saigo type fractional integral operators from our main results in Theorems 4 and 5.

\section{Conflict of Interests}

The authors declare that there is no conflict of interests regarding the publication of this paper.

\section{Acknowledgment}

The authors would like to express their thanks to the referees of the paper for the various suggestions given for the improvement of the paper. 


\section{References}

[1] V. Lakshmikantham and A. S. Vatsala, "Theory of fractional differential inequalities and applications," Communications in Applied Analysis, vol. 11, no. 3-4, pp. 395-402, 2007.

[2] J. D. Ramírez and A. S. Vatsala, "Monotone iterative technique for fractional differential equations with periodic boundary conditions," Opuscula Mathematica, vol. 29, no. 3, pp. 289-304, 2009.

[3] Z. Denton and A. S. Vatsala, "Monotone iterative technique for finite systems of nonlinear Riemann-Liouville fractional differential equations," Opuscula Mathematica, vol. 31, no. 3, pp. 327-339, 2011.

[4] A. Debbouche, D. Baleanu, and R. P. Agarwal, "Nonlocal nonlinear integrodifferential equations of fractional orders," Boundary Value Problems, vol. 2012, article 78, 10 pages, 2012.

[5] H.-R. Sun, Y.-N. Li, J. J. Nieto, and Q. Tang, "Existence of solutions for Sturm-Liouville boundary value problem of impulsive differential equations," Abstract and Applied Analysis, vol. 2012, Article ID 707163, 19 pages, 2012.

[6] Z.-H. Zhao, Y.-K. Chang, and J. J. Nieto, "Asymptotic behavior of solutions to abstract stochastic fractional partial integrodifferential equations," Abstract and Applied Analysis, vol. 2013, Article ID 138068, 8 pages, 2013.

[7] Y. Liu, J. J. Nieto, and Otero-Zarraquiños, "Existence results for a coupled system of nonlinear singular fractional differential equations with impulse effects," Mathematical Problems in Engineering, Article ID 498781, 21 pages, 2013.

[8] P. L. Chebyshev, "Sur les expressions approximatives des integrales definies par les autres prises entre les mêmes limites," Proceedings of the Mathematical Society of Kharkov, vol. 2, pp. 93-98, 1882.

[9] P. Cerone and S. S. Dragomir, "New upper and lower bounds for the Čebyšev functional," Journal of Inequalities in Pure and Applied Mathematics, vol. 3, no. 5, article 77, 2002.

[10] G. Grüss, "Über das Maximum des absoluten Betrages von $(1 /(b-a)) \int_{a}^{b} f(x) g(x) d x-\left(1 /(b-a)^{2}\right) \int_{a}^{b} f(x) d x \int_{a}^{b} g(x) d x, "$ Mathematische Zeitschrift, vol. 39, no. 1, pp. 215-226, 1935.

[11] D. S. Mitrinović, J. E. Pečarić, and A. M. Fink, Classical and New Inequalities in Analysis, vol. 61, Kluwer Academic Publishers Group, Dordrecht, The Netherlands, 1993.

[12] G. A. Anastassiou, Advances on Fractional Inequalities, Springer Briefs in Mathematics, Springer, New York, NY, USA, 2011.

[13] J. Pečarić and I. Perić, "Identities for the Chebyshev functional involving derivatives of arbitrary order and applications," Journal of Mathematical Analysis and Applications, vol. 313, no. 2, pp. 475-483, 2006.

[14] S. Belarbi and Z. Dahmani, "On some new fractional integral inequalities," Journal of Inequalities in Pure and Applied Mathematics, vol. 10, no. 3, article 86, 2009.

[15] Z. Dahmani and L. Tabharit, "Certain inequalities involving fractional integrals," Journal of Advanced Research in Scientific Computing, vol. 2, no. 1, pp. 55-60, 2010.

[16] S. L. Kalla and A. Rao, "On Grüss type inequality for a hypergeometric fractional integral," Le Matematiche, vol. 66, no. 1, pp. 57-64, 2011.

[17] H. Öğünmez and U. M. Özkan, "Fractional quantum integral inequalities," Journal of Inequalities and Applications, vol. 2011, Article ID 787939, 7 pages, 2011.
[18] W. T. Sulaiman, "Some new fractional integral inequalities," Journal of Mathematical Analysis, vol. 2, no. 2, pp. 23-28, 2011.

[19] M. Z. Sarikaya and H. Ogunmez, "On new inequalities via Riemann-Liouville fractional integration," Abstract and Applied Analysis, vol. 2012, Article ID 428983, 10 pages, 2012.

[20] M. Z. Sarikaya, E. Set, H. Yaldiz, and N. Başak, "HermiteHadamard's inequalities for fractional integrals and related fractional inequalities," Mathematical and Computer Modelling, vol. 57, no. 9-10, pp. 2403-2407, 2013.

[21] Z. Dahmani and A. Benzidane, "On a class of fractional qintegral inequalities," Malaya Journal of Matematik, vol. 3, no. 1, pp. 1-6, 2013.

[22] S. D. Purohit and R. K. Raina, "Chebyshev type inequalities for the Saigo fractional integrals and their q-analogues," Journal of Mathematical Inequalities, vol. 7, no. 2, pp. 239-249, 2013.

[23] S. D. Purohit, F. Uçar, and R. K. Yadav, "On fractional integral inequalitiesand their q-analogues," Revista Tecnocientifica URU. In press.

[24] D. Baleanu, S. D. Purohit, and P. Agarwal, "On fractional integral inequalities involving hypergeometric operators," Chinese Journal of Mathematics, vol. 2014, Article ID 609476, 5 pages, 2014.

[25] C. Cesarano, "Identities and generating functions on Chebyshev polynomials," Georgian Mathematical Journal, vol. 19, no. 3, pp. 427-440, 2012.

[26] H. M. Srivastava and H. L. Manocha, A Treatise on Generating Functions, Eliis Horwood Series: Mathematical and Its Applications, Ellis Horwood Ltd., Chichester, UK, 1984.

[27] S. S. Dragomir, "Some integral inequalities of Grüss type," Indian Journal of Pure and Applied Mathematics, vol. 31, no. 4, pp. 397-415, 2000.

[28] Z. Dahmani, O. Mechouar, and S. Brahami, "Certain inequalities related to the Chebyshev's functional involving a RiemannLiouville operator," Bulletin of Mathematical Analysis and Applications, vol. 3, no. 4, pp. 38-44, 2011.

[29] S. D. Purohit and R. K. Raina, "Certain fractional integral inequalities involving the Gauss hypergeometric function".

[30] M. Saigo, "A remark on integral operators involving the Gauss hypergeometric functions," Mathematical Reports of College of General Education, Kyushu University, vol. 11, no. 2, pp. 135-143, $1977 / 78$.

[31] L. Curiel and L. Galué, "A generalization of the integral operators involving the Gauss' hypergeometric function," Revista Técnica de la Facultad de Ingeniería Universidad del Zulia, vol. 19, no. 1, pp. 17-22, 1996.

[32] V. Kiryakova, Generalized Fractional Calculus and Applications, vol. 301 of Pitman Research Notes in Mathematics Series, Longman Scientific \& Technical, Harlow, UK, 1994.

[33] G. E. Andrews, R. Askey, and R. Roy, Special Functions, vol. 71 of Encyclopedia of Mathematics and Its Applications, Cambridge University Press, Cambridge, UK, 1999. 


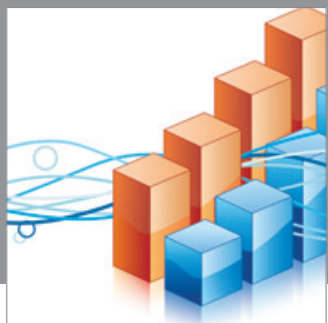

Advances in

Operations Research

mansans

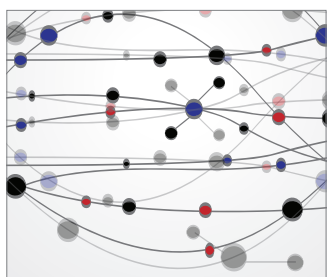

The Scientific World Journal
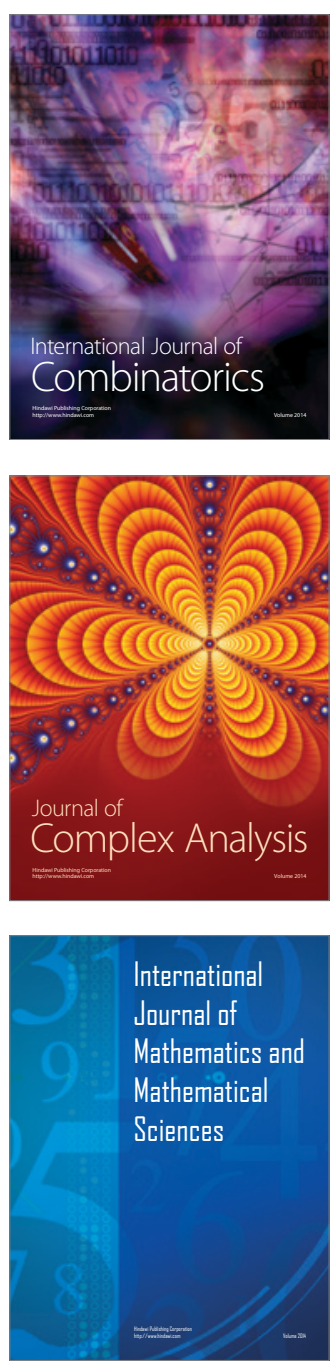
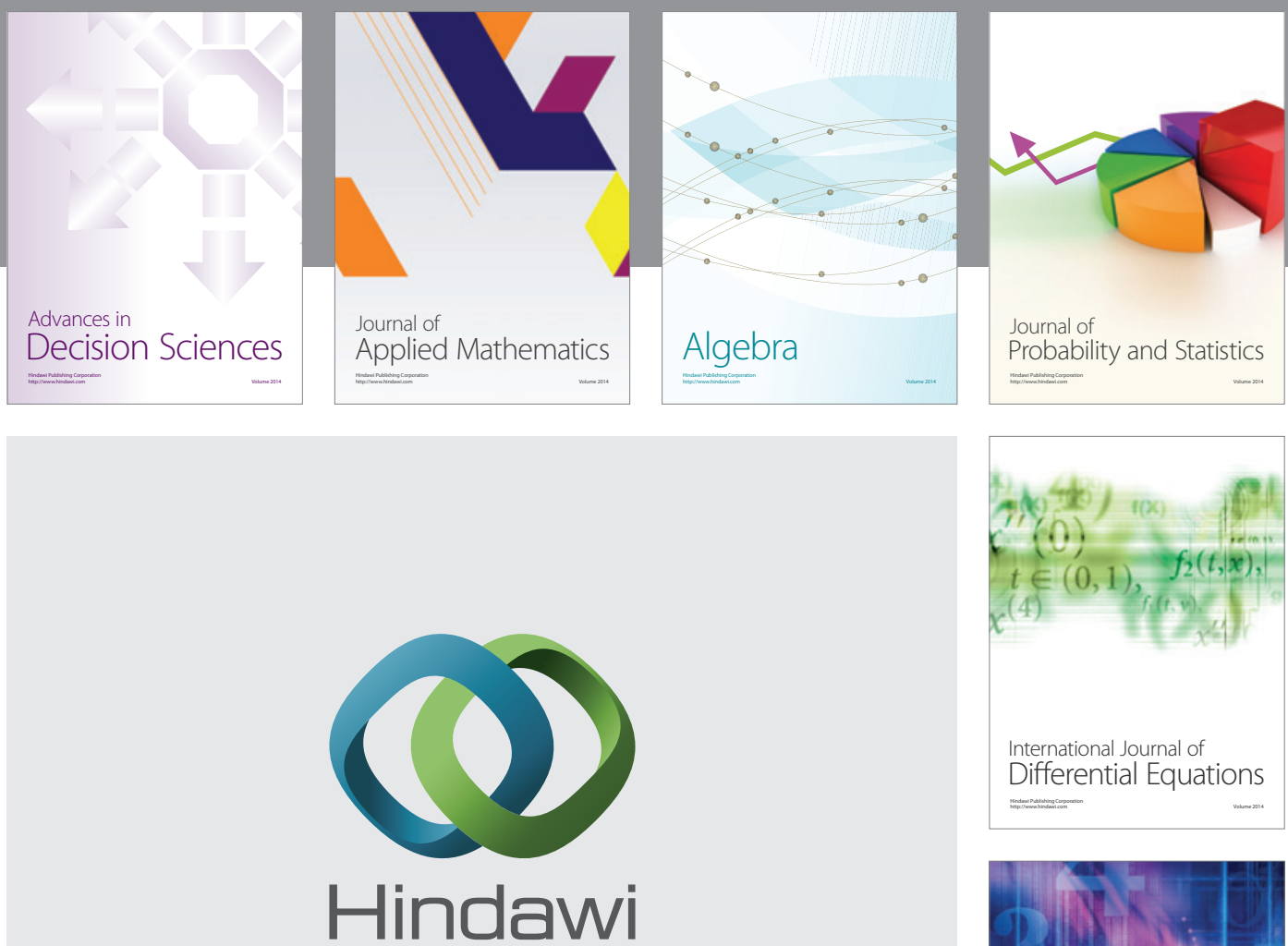

Submit your manuscripts at http://www.hindawi.com
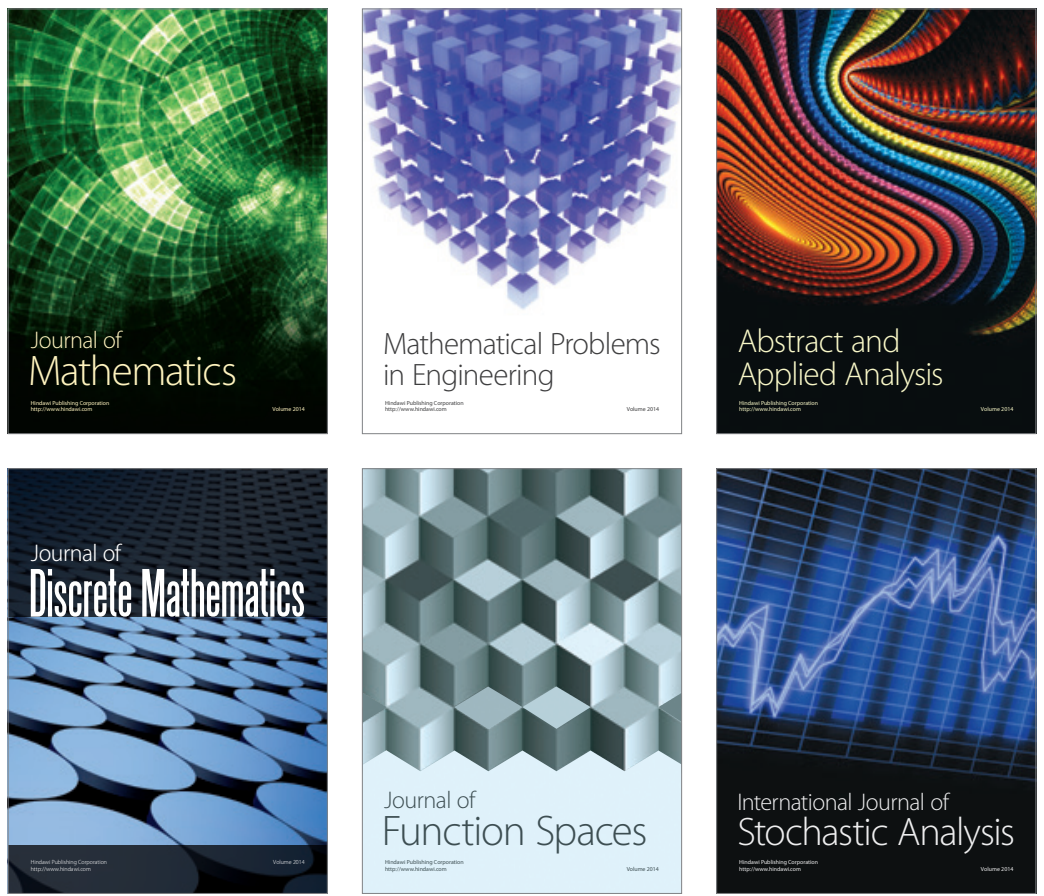

Journal of

Function Spaces

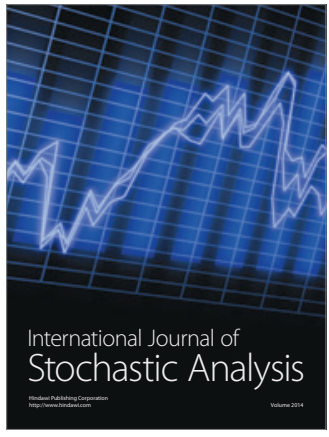

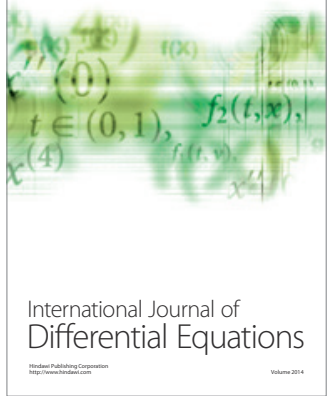
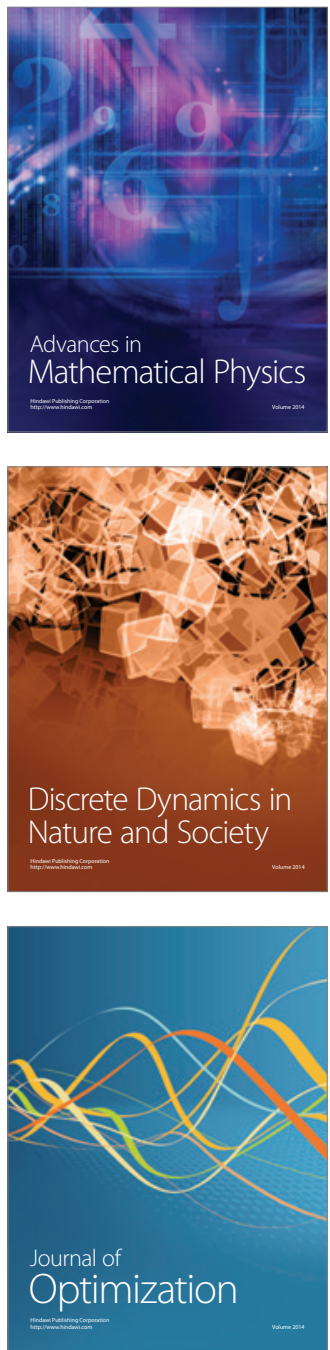